\title{
Investimento reprodutivo e produção de ovos em desovas consecutivas do caranguejo Aratus pisonii (H. Milne Edwards) (Crustacea, Brachyura, Grapsoidea)
}

\author{
Maria Helena de A. Leme \\ Programa de Pós-graduação em Ciências Ambientais, Universidade de Taubaté. Estrada Municipal Dr. José Luiz Cembranelli \\ 5000,12081-010 Taubaté, São Paulo, Brasil.E-mail: mhleme@uol.com.br
}

\begin{abstract}
Reproductive investment and egg production in consecutive spawnings of Aratus pisonii (Crustacea, Brachyura, Grapsoidea). In the present work the egg number laid in sequential broods of the mangrove crab Aratus pisonii (H. Milne Edwards, 1837) were quantified with the aim to test the hypothesis of minus eggs is laid in consecutive spawnings. Ovigerous females of $A$. pisonii were collected in a mangrove forest $\left(23^{\circ} 29^{\prime} \mathrm{S}, 45^{\circ} \mathrm{O} 9^{\prime} \mathrm{W}\right)$ located in Ubatuba, São Paulo State, Brazil from March 2000 to March 2003. In laboratory, these females were categorised in two groups: Group 1, females bearing early-stage eggs and, Group 2, females that accomplished subsequent spawning in laboratory. Both groups of females were analised as number and size of eggs, brood dry weight, individual egg dry weight and reproductive output (ratio of female dry weigh and brood dry weight). Twenty seven females realized a subsequent laid in laboratory. The average time interval between a larval release and the subsequent spawning was of $6.4 \pm 5.6$ days. The number of eggs and the reproductive output did not differ between the both group of females. Slight differences were detected in the size and weight of eggs, being that the eggs oviposited in a subsequent spawning were smaller but heavier than that one observed for Group 1 females. The variability of eggs produced by $A$. pisonii can not be attributed to consecutive spawnings. Perhaps the intrinsic state of females accounts more for those differences in the resource allocation.
\end{abstract}

KEY WORDS. Fecundity; multiple spawning; reproductive effort.

RESUMO. No presente trabalho, o número de ovos postos em desovas seqüenciais pelo caranguejo Aratus pisonii (H. Milne Edwards, 1837) foi quantificado com o objetivo de testar a hipótese de menos ovos serem postos em desovas consecutivas. Fêmeas ovígeras de $A$. pisonii foram coletadas em um manguezal $\left(23^{\circ} 29^{\prime} \mathrm{S}, 45^{\circ} 09^{\prime} \mathrm{W}\right)$ localizado em Ubatuba, SP, Brasil no período de março de 2000 a março de 2003. Em laboratório, essas fêmeas foram separadas em dois grupos: Grupo 1, fêmeas incubando ovos em estágio inicial e, Grupo 2, fêmeas que realizaram desova subseqüente em laboratório. Ambos os grupos de fêmeas foram analisadas quanto ao número e tamanho dos ovos, peso seco da massa de ovos, peso seco do ovo individual e investimento reprodutivo (razão entre o peso seco da fêmea e o peso seco da massa). Vinte e sete fêmeas realizaram nova desova em laboratório. $O$ intervalo médio entre uma liberação larval e uma desova subseqüente foi de $6,4 \pm 5,6$ dias. O número de ovos e o investimento reprodutivo não diferiram entre os grupos de fêmeas. Pequenas diferenças foram detectadas no tamanho e peso dos ovos, sendo que os ovos ovipositados nas desovas seqüenciais foram menores e mais pesados que os observados para as fêmeas do Grupo 1. A variabilidade do número de ovos produzidos por $A$. pisonii não pode ser atribuída a desovas consecutivas. Talvez o estado intrínseco das fêmeas é que some mais por essas diferenças na alocação de recursos.

PALAVRAS-CHAVE. Fecundidade; desovas múltiplas; esforço reprodutivo.

A capacidade de estocar espermas na espermateca é uma das táticas reprodutivas de algumas espécies de braquiúros, a qual permite que várias desovas (extrusão de ovos para os pleópodos) possam ser realizadas dentro de um período de intermuda, sem intervenção de nova cópula (CHeung 1968, Morgan et al. 1983, Hartnoll 1985, Diesel 1989, Sainte-Marie \&
CARRIÈRe 1995). Tanto a realização da desova como os processos envolvidos para tal, como a maturação das gônadas e a manutenção da incubação dos ovos, exigem gastos de energia.

Uma suposição chave em muitos tratamentos de história de vida é que os gastos energéticos alocados para uma reprodução em andamento podem resultar em limitação do crescimento 
da fêmea, devido ao direcionamento de recursos energéticos para fins reprodutivos e não de crescimento, e/ou afetar a fecundidade (número de ovos) da próxima desova, i.e., redução no desempenho reprodutivo (Willians 1966, Calow 1979). Tuomi et al. (1983) relatam duas maneiras pelas quais os animais podem superar essas limitações e estocar energia: uma delas diz respeito à alimentação continuada enquanto o animal está "grávido"; a outra concerne ao intervalo de tempo entre as desovas, de forma que maiores intervalos permitem ao animal obter energia suficiente para ovipositar com sucesso.

Em estudos focando a fecundidade intra-específica em função do tamanho da fêmea, é comum observar uma variabilidade do número de ovos produzidos por indivíduos de uma mesma classe de comprimento. Esta variabilidade torna-se nítida nas plotagens dos dados empíricos em gráficos de dispersão, e nas equações tipo função potência $\left(\mathrm{y}=\mathrm{aX} \mathrm{X}^{\mathrm{b}}\right)$ ou com dados transformados logaritmicamente (regressão linear $\log \mathrm{Y}=\mathrm{a}$ + bLogX), pelas quais podem ser observados baixos valores dos coeficientes de determinação das equações. De maneira geral, essa variabilidade tem sido muitas vezes atribuída à ocorrência de desovas múltiplas; entretanto, essas inferências são especulativas, desde que poucos estudos empíricos têm sido realizados no sentido de quantificar o tamanho da massa ou o número de ovos de desovas sucessivas (veja Bryant \& Hartnoll 1995, SainteMARIE \& CARriÈre 1995). Na prática isto pode ser bastante difícil de ser comprovado para grupos como braquiúros, desde que a retirada de uma massa de ovos do abdômen da fêmea implica no prévio sacrifício desta.

No presente trabalho, foi tentado quantificar o número de ovos postos em desovas seqüenciais pelo caranguejo estuarino Aratus pisonii (H. Milne Edwards, 1837) para testar a hipótese de que menos ovos são colocados em uma desova subseqüente a uma anterior. Para tal, foi assumido que fêmeas ovígeras com ovos em estágio inicial de desenvolvimento e amostradas em campo estariam incubando sua primeira massa de ovos e, fêmeas que realizaram postura em laboratório após prévia liberação larval estariam com sua segunda desova, na verdade com uma desova subseqüente, sem a intervenção de cópula.

\section{MATERIAL E MÉTODOS}

Durante o período de março de 2000 a março de 2003, foram realizadas coletas mensais do caranguejo $A$. pisonii no

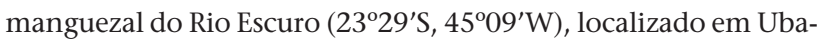
tuba (São Paulo), visando a obtenção de fêmeas ovígeras. Todos os caranguejos foram coletados manualmente tomando-se cuidado particular para evitar danos à massa de ovos no caso do animal estar ovígero. Em laboratório, essas fêmeas foram mensuradas (largura da carapaça, LC) com paquímetro e classificadas em dois grupos de acordo com o estágio de desenvolvimento de seus ovos: Grupo 1, fêmeas incubando ovos em estágio inicial (repletos de vitelo, polo animal ausente), e Grupo 2, fêmeas com ovos em estágio de desenvolvimento avançado, apresentado pigmentação ou com larva totalmente desenvolvida.
As fêmeas do Grupo 1 foram congeladas para análises futuras dos ovos. As fêmeas do Grupo 2 foram mantidas vivas e individualizadas em caixas de polietileno $(11 \times 15$ x $15 \mathrm{~cm})$ contendo seixos e gravetos de árvores de mangues como substrato, preenchidas em parte com água do mar diluída a 20\%o. Alimentação baseada em pedaços de camarão e troca da água foram providenciados todos os dias. Estas fêmeas foram mantidas em uma câmara incubadora tipo B.O.D. com temperatura a $27^{\circ} \mathrm{C}$ e fotoperíodo de verão (14 h claro: $10 \mathrm{~h}$ escuro). Os indivíduos foram inspecionados diariamente para constatação de liberação larval e/ou realização de nova desova em cativeiro. Ocorrência de mudas também foi registrada. Após 30 dias da eclosão e a não ocorrência de nova desova, as fêmeas foram excluídas do experimento.

\section{Tamanho da massa de ovos e investimento reprodutivo}

Todas as fêmeas incubando ovos em estágio inicial foram analisadas quanto ao número de ovos (fecundidade, F) e investimento reprodutivo (IR), incluindo as que realizaram nova desova em laboratório (Grupo 2).

Para estimar a fecundidade, os pleópodos das fêmeas foram dissecados e os ovos dissociados com auxílio de pinças e pincel. Após rápida secagem com papel de filtro, toda a massa de ovos foi então pesada (peso úmido da massa de ovos, PUMO) em balança digital eletrônica $(0,1 \mathrm{mg})$. Três subamostras foram separadas, pesadas e o número de ovos contados sob estereomicroscópio. Tanto as fêmeas como suas respectivas massas de ovos foram colocadas para secar em uma estufa a $60^{\circ} \mathrm{C}$ por aproximadamente $48 \mathrm{~h}$, após o que foram imediatamente pesadas novamente.

A fecundidade foi estimada dividindo o peso seco da massa total de ovos (PSMO; incluindo o peso das subamostras retiradas) pelo peso seco das subamostras e então multiplicando esse valor pelo número médio de ovos contado previamente das subamostras. O peso seco dos ovos individuais (PSO) foi estimado dividindo o peso seco da massa de ovos pelo número de ovos da massa total.

O tamanho dos ovos foi determinado mensurando uma subamostra de 10 ovos de cada fêmea antes desta ser congelada para as análises da fecundidade. Estes ovos foram mensurados no seu diâmetro sob uma ocular micrométrica acoplada a um estereomicroscópio óptico. A partir do diâmetro médio dos ovos calculou-se o volume considerando-se a forma esférica dos ovos em estágio inicial.

O investimento reprodutivo (IR) foi estimado dividindo o peso seco da massa de ovos (PSMO) pelo peso seco da fêmea (PSF) e então multiplicando-se por 100 para obtenção de valores percentuais (HINEs 1982).

Regressões foram estabelecidas para as relações entre F e PSMO vs. LC, após transformações logarítmicas (ln) dos dados para obtenção da equação linear $(\ln Y=a+b \ln X)$. Análises de covariância (ZAR 1999) foram aplicadas para comparar os coeficientes de regressão (b) e os interceptos (a) entre regressões equivalentes para os grupos de fêmeas, mantendo-se a LC como 
covariável. Quando os coeficientes de regressão não diferem, significa que as linhas das regressões, são paralelas e originadas de uma mesma população amostral, enquanto que diferenças nos interceptos indicam variação da fecundidade absoluta (Somers 1991), ou no caso do presente trabalho, do peso da massa de ovos. Quando os coeficientes de regressão não diferiram estatisticamente, calculou-se o coeficiente de regressão comum (bc) e estimou-se o novo intercepto para o conjunto total dos dados (ZAR 1999). Dessa forma a hipótese nula $\left(\mathrm{H}_{0}\right)$ corresponde à não diferença no número de ovos e no peso da massa de ovos entre as desovas analisadas.

As variáveis LC, IR, peso dos ovos, diâmetro e volume dos ovos foram comparadas para os diferentes eventos de desova por meio de teste $t$ para amostras independentes. Neste caso, a hipótese nula significa que tais variáveis não diferem entre as desovas observadas.

\section{RESULTADOS}

\section{Número de ovos em desovas consecutivas}

Um total de 154 fêmeas ovígeras foram monitoradas para verificação da realização de nova postura em laboratório, sendo que destas, apenas 27 fêmeas realizaram nova postura após prévia liberação de suas larvas. De maneira geral, dois eventos principais (excetuando-se a morte da fêmea) ocorreram logo após a liberação das larvas da desova anterior: nova postura (média $=6,4 \pm 5,6$ dias) ou muda (média $=13,7 \pm 8,8$ dias). As desovas seqüenciais ocorreram em períodos de tempo variando de 3 a 21 dias.

A distribuição de freqüência em tamanho das fêmeas analisadas, tanto do Grupo $1(\mathrm{~N}=40)$ como do Grupo $2(\mathrm{~N}=$ 27), é apresentada na figura 1. Fêmeas menores (16 a 20 mmLC) foram mais representativas na população perfazendo aproximadamente $70 \%$ da população amostral. A freqüência de fêmeas que realizaram desova sucessiva foi similar entre todas as classes de largura de carapaça analisadas, de forma que, não houve diferença estatística do tamanho das fêmeas analisadas para ambos os grupos (Teste-t, p > 0,05) (Tab. I).

As equações obtidas por meio das regressões lineares das variáveis LC contra F e PSMO, para fêmeas do Grupo 1 e 2, são apresentadas na tabela II. Os coeficientes de regressão e os interceptos dessas regressões não diferiram estatisticamente

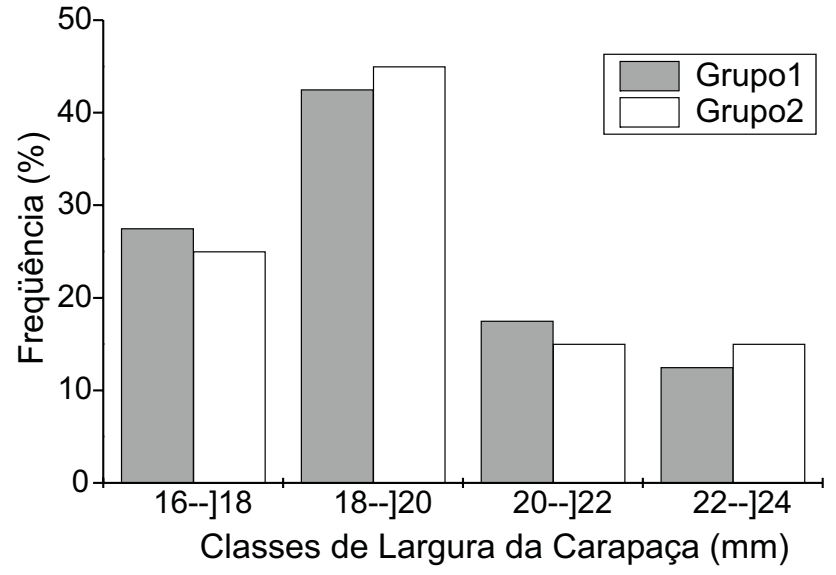

Figura 1. Aratus pisonii. Distribuição da freqüência relativa de fêmeas ovígeras dos Grupos 1 e 2 nas classes de largura da carapaça.

(ANCOVA, p > 0,05), demonstrando que o número de ovos produzidos em desovas seqüenciais pode ser tão elevado quanto em uma desova anterior. Recalculando-se o novo valor dos interceptos a partir do coeficiente de regressão comum ( $b c=2,816$ ), obteve-se diferença estatística significativa entre os interceptos da regressão da fecundidade contra a largura da carapaça, indicando que, de fato, a segunda postura pode ser significativamente maior que a primeira observada (ANCOVA, $\mathrm{F}=80,36$, $\mathrm{p}<0,0001)$. O mesmo foi obtido para a relação LC x PSMO, aplicando-se o coeficiente de regressão comum, bc $=2,838$ (ANCOVA, F = 70,82, $\mathrm{p}<0,0001$ ).

\section{Investimento reprodutivo nas novas posturas}

Estimativas do IR e da fecundidade originados das fêmeas ovígeras coletadas em campo (Grupo 1) e das que realizaram nova postura em laboratório (Grupo 2) apresentaram resultados semelhantes (Tab. I). O IR não diferiu significativamente entre as fêmeas do Grupo 1 e 2 (Teste-t, $t=0,007$, g.l. = 65, p = 0,99) e não houve correlação entre IR e tamanho da fêmea (Correlação linear de Pearson, $r=0,027, p=0,83$ ). Entretanto, parece haver uma tendência para uma maior homogeneidade do investimento reprodutivo em fêmeas maiores que $20 \mathrm{~mm}$ de LC, como pode ser observado na figura 2, refletindo também em uma menor variação do número de ovos produzidos por fêmeas maiores.

Tabela I. Aratus pisonii. Médias ( \pm DP) e variações do investimento reprodutivo (IR), da fecundidade (F) e do tamanho das fêmeas (LC) do Grupo 1 e do Grupo 2. (N) Número de fêmeas analisadas em cada grupo.

\begin{tabular}{|c|c|c|c|c|c|}
\hline \multicolumn{2}{|l|}{ Fêmeas } & IR (\%) & $\mathrm{F}$ & $\mathrm{LC}(\mathrm{mm})$ & \multirow{2}{*}{$\frac{N}{40}$} \\
\hline Grupo 1 & $M( \pm D P)$ & $15,1 \pm 4,5$ & $14978 \pm 6105$ & $19,2 \pm 2,0$ & \\
\hline & Amplitude & $4,8-22,1$ & $6338-31839$ & $16,1-23,6$ & \\
\hline \multirow[t]{2}{*}{ Grupo 2} & $M( \pm D P)$ & $15,1 \pm 3,5$ & $14298 \pm 4484$ & $19,5 \pm 1,8$ & 27 \\
\hline & Amplitude & $7,2-22,7$ & $7601-23672$ & $16,7-22,8$ & \\
\hline Média Total & $M( \pm D P)$ & $15,1 \pm 4,1$ & $14704 \pm 5483$ & $19,3 \pm 1,9$ & 67 \\
\hline
\end{tabular}


Tabela II. Aratus pisonii. Equações das regressões da fecundidade (F) e do peso seco da massa de ovos (PSMO) contra a largura da carapaça (LC), obtidas para as fêmeas do Grupo 1 e do Grupo 2. $\left(r^{2}\right)$ Coeficiente de determinação, valores de $F$ (teste).

\begin{tabular}{|c|c|c|c|c|c|}
\hline Fêmeas & LC vs. & $N$ & Modelo da regressão $(y=a+b x)$ & $r^{2}$ & Teste F \\
\hline \multirow[t]{2}{*}{ Grupo 1} & $\mathrm{~F}$ & 40 & $\operatorname{Ln} F=0,744+2,983 \ln \mathrm{LC}$ & 0,58 & $52,8^{* \star *}$ \\
\hline & PSMO (mg) & 36 & Ln PSMO $=-4,472+2,99 \ln \mathrm{LC}$ & 0,56 & $43,6^{* * *}$ \\
\hline \multirow[t]{2}{*}{ Grupo 2} & $\mathrm{~F}$ & 27 & $\operatorname{Ln} F=2,040+2,520 \ln \mathrm{LC}$ & 0,49 & $26,3^{* * *}$ \\
\hline & PSMO (mg) & 27 & Ln PSMO $=-3,317+2,587 \ln \mathrm{LC}$ & 0,51 & $25,9 * * *$ \\
\hline
\end{tabular}

${ }^{* * *}$ Coeficientes de regressão diferentes de zero: $\left({ }^{*}\right) p<0,05 ;\left({ }^{* *}\right) p<0,01 ;\left(^{* * *}\right) p<0,001$, ns. $=p>0,05 ;$ ANCOVA.

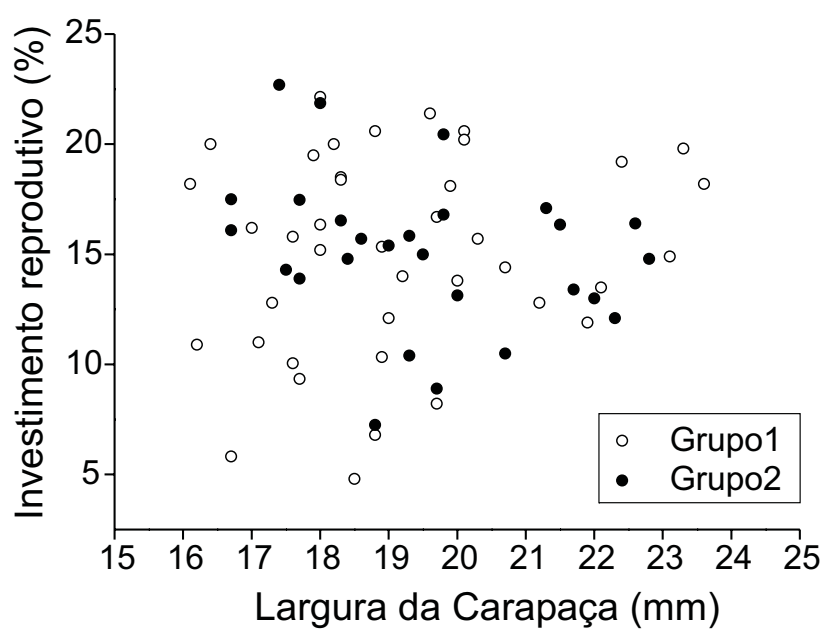

Figura 2. Aratus pisonii. Relação entre tamanho e investimento reprodutivo determinado para fêmeas ovígeras do Grupo 1 e Grupo 2.

\section{Tamanho e peso dos ovos}

O diâmetro médio dos ovos em estágio de desenvolvimento inicial das 67 fêmeas ovígeras analisadas no total foi de $0,30 \pm 0,01 \mathrm{~mm}$, com diâmetros variando de 0,26 a 0,34 mm. O volume correspondente foi de $0,014 \pm 0.002 \mathrm{~mm}^{3}$ com amplitude de 0,010 a $0,020 \mathrm{~mm}^{3}$.

O tamanho dos ovos da espécie $A$. pisonii, analisado tanto em diâmetro como em volume, diferiu estatisticamente entre as fêmeas do Grupo 1 e do Grupo 2 (teste-t, p < 0,05) (Tab. III). O diâmetro dos ovos das fêmeas do Grupo 2 foram 4,4\% menores que os das fêmeas do Grupo 1 (Teste $t, p=0,0007$ ), o que corresponde a 14,6\% de diferença em volume. Essa diferença, de certa forma, já era esperada, pois, assim que notou-se a nova postura no abdome das fêmeas do Grupo 2, os ovos foram imediatamente mensurados. Ao contrário, fêmeas capturadas em campo, podiam estar com a massa de ovos há algum tempo e, portanto, já mostrar evidências de aumento em tamanho devido às condições presentes em campo.

A biomassa individual dos ovos em estágio inicial apresentou valor médio de 5,61 $\pm 0,35 \mu$ e amplitude de 4,8 a 6,52 $\mu \mathrm{g}$. Ovos referentes à segunda desova foram $4,7 \%$ mais pesados e diferiram significativamente dos ovos das fêmeas do Grupo 1 (Teste $t, p=0,003$ ) (Tab. III).
Tabela III. Aratus pisonii. Diâmetro, volume e peso médios ( \pm DP) dos ovos em estágio inicial, verificados nas fêmeas ovígeras do Grupo 1 e Grupo 2.

\begin{tabular}{ccccc}
\hline Fêmeas & $\begin{array}{c}\text { Diâmetro } \\
(\mathrm{mm})\end{array}$ & $\begin{array}{c}\text { Volume } \\
\left(\mathrm{mm}^{3}\right)\end{array}$ & $\begin{array}{c}\text { Peso do Ovo } \\
(\mu \mathrm{g})\end{array}$ & $\mathrm{N}$ \\
\hline Grupo 1 & $0,305 \pm 0,017$ & $0,0149 \pm 0,002$ & $5,50 \pm 0,36$ & 40 \\
Grupo 2 & $0,292 \pm 0,011$ & $0,0130 \pm 0,001$ & $5,76 \pm 0,26$ & 27 \\
\hline
\end{tabular}

\section{DISCUSSÃO}

A espécie $A$. pisonii realiza mais de uma postura em um período de intermuda, sem a necessidade de nova cópula. Estas novas desovas podem ser realizadas em intervalos de tempo tão curtos quanto três dias após a eclosão das larvas da desova anterior. Provavelmente, após uma segunda desova, ou mais (o número de desovas que a espécie pode realizar em uma intermuda ainda é incerta), o animal realiza a muda. Morgan et al. (1983) demonstraram que o caranguejo grapsóide Rhithropanopeus harrisii (Gould, 1841) é capaz de desovar até quatro vezes sem intervenção de cópula, entretanto, o número de desovas pode ser ainda maior em outras espécies chegando a 13, como verificado para Menippe mercenaria (Say, 1818) (Cheung 1968).

O intervalo de tempo entre uma desova e outra pode ser bastante variável entre as diferentes espécies de crustáceos. Esse intervalo varia em função do tempo de incubação de uma massa de ovos e do período necessário à restauração das gônadas (SudHA \& ANILKUMAR 1996), podendo variar ainda em função da idade do animal (ATTARd \& Hudon 1987). Uma substancial quantidade de lipídios e proteínas é necessária para restauração gonadal de forma que o comportamento de forrageamento das fêmeas durante uma reprodução em andamento pode ser um fator limitante para a produção de uma nova desova (WEAR 1974, TuOMI et al. 1983, HenMi 2003).

Tanto o crescimento como a reprodução são processos que competem por recursos, logo, podem envolver riscos (RofF 1983, Hartnoll 1985). Para braquiúros, crescimento requer recurso ou reserva energética antes da ecdise e também para completar o crescimento dos tecidos durante a intermuda. Tanto a aquisição de recursos como o próprio ato da muda envolve riscos de mortalidade, o que pode reduzir a futura participação reprodutiva (HARTNOLl 1985). Dessa forma, as variações de tempo, entre os eventos muda e reprodução, permitem ajustes no 
nível de gasto de energia alocado para reprodução em conjunção com diminuição nas taxas de crescimento conforme as fêmeas crescem (BRody 1991).

PianKa \& Parker (1975) definem o esforço reprodutivo como a proporção de recursos em um organismo que é alocado para reprodução em algum tempo determinado ou por algum intervalo de tempo. Para braquiúros, desovas múltiplas parecem ser estratégias que maximizam a produção sazonal de ovos e, quando realizadas em curto intervalo de tempo minimizam, também, o tempo necessário para a produção de juvenis em uma estação do ano (OH \& HarTNOll 1999). Entretanto, existe uma inter-relação entre o esforço reprodutivo corrente e a futura produção de proles (Williams 1966, Pianka \& Parker 1975, Calow 1979). De acordo com a teoria, um aumento no esforço reprodutivo corrente pode diminuir o futuro potencial reprodutivo por afetar tanto o crescimento do animal, como a fecundidade subseqüente.

GILLESPIE (1977) chama a atenção quanto à importância de se compreender o significado adaptativo das estratégias de vida dando-se maior atenção aos componentes de variância do número de proles. No caso do caranguejo A. pisonii, uma das causas da variância pode estar relacionada à sua capacidade de realizar desovas consecutivas, entretanto, o tamanho da massa de ovos deve depender principalmente do estado intrínseco da fêmea. Maior variação do número de ovos ocorreu em fêmeas menores que $20 \mathrm{~mm}$ de largura de carapaça, as quais predominam na população, e para as quais o investimento reprodutivo se mostra bastante variável (4,8 a 22,7\%). Uma possível explicação para isso é que fêmeas menores, que mudam com mais freqüência, também têm que utilizar grande parte de energia para alocar em crescimento somático (ATTARd \& Hudon 1987, Brody 1991).

Estudos sobre as inter-relações fecundidade e idade, ou tamanho, no caso de braquiúros, são bastante comuns, entretanto, mensurações sobre o investimento reprodutivo em diversas massas de ovos do mesmo indivíduo é mais complicado (LAwlor 1976). Para A. pisonii, o investimento reprodutivo obtido a partir das desovas subseqüentes, realizadas sob condições controladas em laboratório, foi similar ao obtidos de fêmeas ovígeras coletadas em campo, chamadas neste estudo de Grupo 1. Apesar de não ser possível assegurar que estas fêmeas do Grupo 1 estivessem realmente incubando sua primeira massa de ovos, os resultados apresentaram variações similares do IR nas desovas subseqüentes realizadas em laboratório.

A mesma similaridade foi observada com relação ao número de ovos postos nas novas desovas, constatando-se que, além destas poderem ser tão fecundas quanto a primeira, elas são significativamente maiores $(\mathrm{p}<0,0001)$, contribuindo, dessa forma, para a elevada produção de ovos durante a estação reprodutiva que ocorre na primavera e verão (LEME 2002). Maior produção de ovos em desovas subseqüentes em relação à anterior tem sido relatada para algumas espécies de braquiúros (Bryant \& Hartnoll 1995, Sainte-Marie \& Carrière 1995) e para o Isopoda Armadillidium vulgare (Latreille, 1804) (LAWLOR 1976).

De acordo com Hartnoll \& Gould (1988), a combinação entre crescimento e reprodução que maximizará a produção de proles no tempo de vida é determinada pelo perfil de mortalidade das espécies. A espécie $A$. pisonii apresenta elevada taxa de mortalidade larval (WARNER 1967) e tempo de vida estimado em um ou dois anos (LEME 2002). Os resultados obtidos no presente trabalho complementam as informações sobre a estratégia de vida de $A$. pisonii, indicando que a espécie otimiza a produção de ovos por estação reprodutiva por meio de desovas consecutivas em um único período de intermuda. Tanto a otimização na produção de ovos como a realização de muda dentro da estação reprodutiva são fatores que exigem um elevado gasto energético, podendo causar alta taxa de mortalidade adulta no final do período reprodutivo (Williams 1966, Leme 2002). Esta associação negativa entre investimento reprodutivo e tempo de vida da população parental é alvo de diversas discussões teóricas sobre estratégias de história de vida (CALOW 1979), envolvendo organismos que apresentam semelparidade (i.e., um evento reprodutivo ao longo da vida) e iteroparidade (i.e., várias desovas ao longo da vida) (Cole 1954). A espécie A. pisonii apresenta como características de história de vida a iteroparidade e crescimento indeterminado, constituindo-se em um elemento de estudos bastante interessante para abordagens de melhor conhecimento da evolução de tendências adaptativas de história de vida.

Como conclusão deste trabalho, pode-se inferir que a espécie $A$. pisonii devota muita energia para a produção de uma desova subseqüente à uma anterior, possivelmente às custas de seu próprio valor reprodutivo residual, i.e. poucas fêmeas sobreviverão a uma segunda estação reprodutiva. Fatores como densidade, sasonalidade reprodutiva e heterogeneidade ambiental podem estar envolvidos na evolução dos mecanismos fisiológicos dos pais que garantem adaptabilidade da progênie e o sucesso reprodutivo, visualizado como indivíduos que atingem a maturidade sexual garantindo a sobrevivência da espécie.

Dessa forma, o estado intrínseco da fêmea parece ser o principal determinante da quantidade de energia investida para reprodução (Roff 1983) com impacto direto na fecundidade da população (WenNer 1977, BROdy 1991, HeNMi 2003).

\section{AGRADECIMENTOS}

À Fundação de Amparo à Pesquisa do Estado de São Paulo pelo auxílio financeiro (JP \# 99/10267-5 and \# 99/13030-6). Ao Departamento de Biologia e à Pró-Reitoria de Pesquisa e Pós-Graduação da Universidade de Taubaté e aos professores do Laboratório de Zoologia Itamar A. Martins e Valter J. Cobo.

\section{REFERÊNCIAS BIBLIOGRÁFICAS}

Attard, J. \& C. Hudon. 1987. Embryonic development and energetic investment in egg production in relation to size female lobster (Homarus americanus). Canadian Journal of Fisheries and Aquatic Sciences, Ottawa, 44: 1157-1164.

Brody, M.S. 1991. Variation in reproductive output of the isopod, Armadillidium vulgare, p. 77-113. In: A. WenNER \& A.

Revista Brasileira de Zoologia 23 (3): 727-732, setembro, 2006 
KuRIS (Eds). Crustacean egg production. Rotterdam, A.A. Balkema, XII+401p.

Bryant, A.D. \& R.G. Hartnoll. 1995. Reproductive investment in two spider crab with different breeding strategies. Journal of Experimental Marine Biology and Ecology, Amsterdam, 188: $261-275$.

Calow, P. 1979. The cost of reproduction - a physiological approach. Biological Reviews, Cambridge, 54: 23-40.

Cheung, T.S. 1968. Transmolt retention of sperm in adult female stone crab, Menippe mercenaria (Say). Crustaceana, Leiden, 15: 117-120.

Cole, L.C. 1954. The population consequences of life history phenomena. Quarterly Review of Biology, Chicago, 29: 103-137.

DiesEL, R. 1989. Structure and function of the reproductive system of the symbiotic spider crab Inachus phalangium (Decapoda: Majidae) observations on sperm transfer, sperm, storage and spawning. Journal of Crustacean Biology, Lawrence, 9: 266277.

GILLESPIE, J.H. 1977. Natural selection for variances in offspringer number: a new evolutionary principle. American Naturalist, Chicago, 111: 1010-1014.

HarTNOLL, R.G. 1985. Growth, sexual maturity and reproductive output, p. 101-128. In: A. M. Wenner (Ed.). Factors in adult growth. Rotterdam, A.A. Balkema. XIII + 362 .

Hartnoll, R.G. \& P. Gould. 1988. Brachyuran life history strategies and the optimization of egg production. Symposium of the Zoological Society of London, London, 59: 1-9.

Henmi, Y. 2003. Trade-off between brood size and brood interval and the evolution of underground incubation in three fiddler crabs (Uca perplexa, U. vocans, and U. dussumieri). Journal of Crustacean Biology, Lawrence, 23: 46-54.

Hines, A.H. 1982. Allometric constraints and variables of reproductive effort in brachyuran crabs. Marine Biology, Berlin, 69: 309-320.

LAWLOR, L.R. 1976. Molting, growth and reproductive strategies in the terrestrial isopod, Armadillidium vulgare. Ecology, Durham, 57: 1179-1194.

LEME, M.H. DE A. 2002. A comparative analysis of the population biology of the mangrove crabs Aratus pisonii and Sesarma rectum (Brachyura, Grapsidae) from north coast of São Paulo State, Brazil. Journal of Crustacean Biology, Lawrence, 22 (3): 553-557.

Recebido em 21.X.2005; aceito em 22.VIII.2006.
Morgan, S.G.; J.W. Goy \& J.D. Costlow Jr. 1983. Multiple ovipositions from single matings in the mud crab Rhithropanopeus harrisii. Journal of Crustacean Biology, Lawrence, 3 (4): 542-547.

OH, C.W. \& R.G. HARTNOLl. 1999. Size at maturity, reproductive output, and seasonal reproduction of Philocheras trispinosus (Decapoda) in Port Erin Bay, Isle de Man. Journal of Crustacean Biology, Lawrence, 19 (2): 252-259.

Pianka, E.R. \& W.S. Parker. 1975. Age-specific reproductive tactics. American Naturalist, Chicago, 109 (968): 453-464.

Roff, D.A. 1983. An allocation model of growth and reproduction in fish. Canadian Journal of Fisheries and Aquatic Sciences, Ottawa, 40: 1395-1404.

Sainte-Marie, B. \& C. Carrière. 1995. Fertilization of the second clutch of eggs of snow crab, Chionoecetes opilio, from females mated once or twice after their molt to maturity. Fishery Bulletin, Oxford, 93: 759-764.

SOMERS, K.M. 1991. Characterizing size-specific fecundity in crustaceans, p. 357-378. In: A. Wenner \& A. KurIs (Eds). Crustacean egg production. Rotterdam, A.A. Balkema, XII+401p.

SudHA, K. \& G. ANILKUMAr. 1996. Seasonal growth and reproduction in a highly fecund brachyuran crab, Metopograpsus messor (Forskål) (Grapsidae). Hydrobiologia, Netherlands, 319: 1521.

Tuomi, J.; T. Hakala \& E. Haukioja. 1983. Alternative concepts of reproductive effort, costs of reproduction, and selection in life-history evolution. American Zoologist, Thousand Oaks, 23: 25-34.

WARNER, G.F. 1967. The life history of the mangrove tree crab, Aratus pisoni. Journal of Zoology, London, 153: 321-335.

WEAR, R.G. 1974. Incubation in British decapod Crustacea, and the effects of temperature on the rate and success of embrionic development. Journal of Marine Biological Association of United Kingdom, London, 54: 745-762.

Wenner, A.M. 1977. Food supply, feeding habits, and egg production in Pacific mole crabs (Hippa pacifica Dana). Pacific Science, Honolulu, 31: 39-47.

Williams, G.C. 1966. Natural selection, the costs of reproduction, and a refinement of Lack's principle. American Naturalist, Chicago, 100: 687-690.

ZAR, J. H. 1999. Biostatistical analysis. Upper Saddle River, Prentice-Hall, 663p. 